\title{
Encoding of macroscopic second-order non-linearity via all-optical polar alignment in substituted norbornene polymer thin films
}

\author{
Victor M. Churikov ${ }^{\mathrm{a}, 1}$, Meng-Feng Hung ${ }^{\mathrm{a}}$, Chia-Chen Hsu ${ }^{\mathrm{a}, *}$, \\ Chung-Wai Shiau ${ }^{b}$, Tien-Yau Luh ${ }^{\mathrm{b}}$ \\ a Department of Physics, National Chung Cheng University, Ming Hsiung, Chiayi 621, Taiwan, ROC \\ b Department of Chemistry, National Taiwan University, Taipei, Taiwan, ROC
}

Received 26 September 2000; in final form 23 October 2000

\begin{abstract}
Efficient all-optical poling of substituted norbornene azo-molecules-doped polymer thin films is presented. The growth and decay of photoinduced second-order susceptibility $\chi^{(2)}$ has been studied. The possibility of encoding $\chi^{(2)}$ at low seeding intensities (about $10 \mathrm{MW} / \mathrm{cm}^{2}$ for fundamental and $100 \mathrm{~W} / \mathrm{cm}^{2}$ for second harmonic) is demonstrated. The value of encoded $\chi^{(2)}$ in mono-norbornene-doped polymethylmethacrylate (PMMA) and poly-norbornene polymer have been found to be with the same order of magnitude despite the latter revealed considerably higher first hyperpolarizability. This is due to the rigidity of the poly-norbornene polymer backbone and the strong orientational correlation between pendant chromophores that reduce reorientation of molecules. (c) 2000 Elsevier Science B.V. All rights reserved.
\end{abstract}

Non-linear optical azo-dye chromophores are attractive candidates for applications in harmonic generation, electro-optics and information storage devices [1]. Large second-order non-linearities of azo-dye molecules allow us to attain high macroscopic second-order susceptibility $\chi^{(2)}$ via variety of poling techniques. Optical poling method is of particular interest because of its simplicity, efficiency and possibility of automatic satisfying the phase matching condition for second harmonic

\footnotetext{
${ }^{*}$ Corresponding author. Fax: +886-5-272-0587.

E-mail address: cchsu@phy.ccu.edu.tw (C.-C. Hsu).

${ }^{1}$ Permanent address: South Ural University, Nonlinear Optics Laboratory, 76, Lenina Ave, Chelyabinsk, 454080, Russian Federation.
}

(SH) generation. The mechanism of all-optical poling of azo-molecules can be briefly summarized as follows [2]. The azo-molecules can come in stable trans form and excited cis form. If the medium is exposed to a sum of mutually coherent optical waves with frequencies $\omega$ and $2 \omega$, then the probability of excitation of a given molecule $P(\theta)$ is contributed from following three terms: $a_{1}\left|E_{2 \omega}^{2}\right| \cos ^{2} \theta$, associated with one-photon absorption of $\mathrm{SH}, a_{2}\left|E_{\omega}^{4}\right| \cos ^{4} \theta$, associated with two-photon absorption of fundamental, and interference term $a_{3}\left|E_{\omega}^{2} E_{2 \omega}^{*}\right| \cos \left(\Delta k z+\left(\phi_{2 \omega}-2 \phi_{\omega}\right)\right) \cos ^{3} \theta$. Here $E_{\omega}$ and $E_{2 \omega}$ are electric field vectors of the optical waves, coefficients $a_{1}, a_{2}, a_{3}$ depend on the transition dipole moment and the difference between the dipole moments in the excited and 
ground states, $\theta$ is the angle between polar axis and the dipole moment of the molecule, $z$ is the propagation direction, $\Delta k=k_{2 \omega}-2 k_{\omega}$ is a wave vector mismatch and $\phi_{\omega}, \phi_{2 \omega}$ are initial phases for the fundamental and SH waves, respectively. The last term possesses a polar asymmetry and is supposed to be responsible for creation of the noncentrosymmetry in an originally isotropic medium. Under selective polar excitation the molecules with dipole moments parallel to the polarity of the interference term will experience trans-cis isomerization followed by reverse cis-trans reaction with change of previous orientation. This finally results in a net orientation and quasi-permanent noncentrosymmetry.

A detailed theoretical consideration of photoinduced molecular polar alignment was presented in $[3,4]$. It has been shown that relaxation of photoinduced $\chi^{(2)}$ can be described by a bi-exponential function [4]

$\chi^{(2)}(t)=A_{1} \exp \left(-t / \tau_{A_{1}}\right)+A_{2} \exp \left(-t / \tau_{A_{2}}\right)$,

the faster decay component is attributed to the change of cis population and the second component is due to change of induced polar order [4].
The most well-studied azo-dye material for alloptical poling is disperse red 1 (DR1) in polymethylmethacrylate (PMMA). The $\chi^{(2)}$ value as large as $140 \mathrm{pm} / \mathrm{V}$ (comparable with standard corona poling) was reported for a spin-coated DR1MMA co-polymer (side-chain system) [2]. The $\chi^{(2)}$ achieved in a $10 \%$ DR1-PMMA guest-host system has been found to be 1 order of magnitude smaller. The intensities of $3 \mathrm{GW} / \mathrm{cm}^{2}$ for fundamental and $30 \mathrm{MW} / \mathrm{cm}^{2}$ for seeding SH were used for poling of the thin film samples [2].

In this Letter we present efficient optical poling of substituted norbornene azo-molecule polymer thin films. Recently hyper-Rayleigh scattering (HRS) study of substituted norbornene chromophores has shown that they possess large values of the first hyperpolarizability $\beta[5,6]$. Moreover, in comparison with DR1, the substituted norbornene molecules possess blue-shifted absorption peak and considerably smaller absorption at the $\mathrm{SH}$ wavelength of $\mathrm{Nd}$ :YAG laser, that makes them attractive for non-linear optical applications.

The structure and absorption spectra of the samples studied are shown in Fig. 1. The guesthost systems were made by dissolving DR1 or

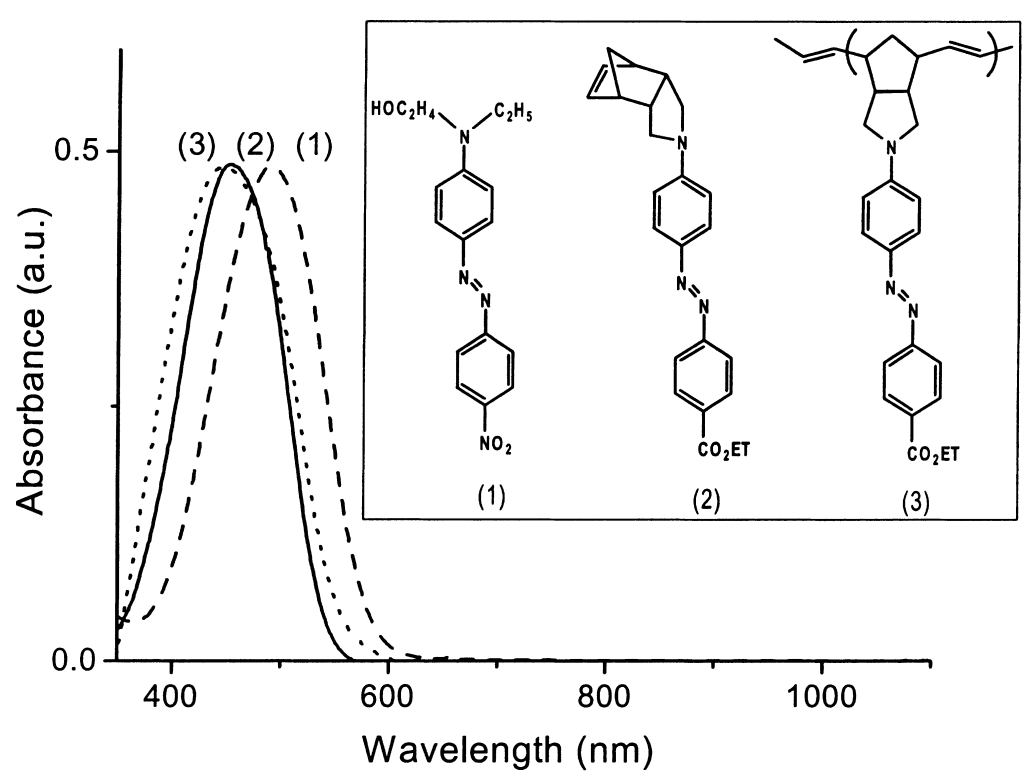

Fig. 1. Absorption spectra of thin films of DR1-PMMA (dashed line), mono-norbornene-PMMA (solid line) and poly-norbornene (dot line). The inset shows the molecular structures of the constituted dye molecules in these three polymer systems. 
mono-norbornene dyes together with PMMA in chloroform. The weight concentration was $10 \%$ for both DR1 and mono-norbornene dopants. The poly-norbornene sample was formed by dissolving of pure polymer molecules in chloroform. Thin films were prepared by spin coating solutions onto glass substrates. The remaining solvent was evaporated in dry chamber during several hours. The mono- and poly-norbornene thin films were 1.1 and $0.1 \mu \mathrm{m}$ thick and their optical densities at 532 $\mathrm{nm}$ were 0.13 and 0.03 , respectively. The thickness and optical density of the DR1-PMMA sample were $0.8 \mu \mathrm{m}$ and 0.45 , respectively.

A Q-switched Nd:YAG laser with 7 ns pulse width and $10 \mathrm{~Hz}$ repetition rate was used as the light source. The seeding SH was generated by a $\beta$ barium borate (BBO) crystal. In the poling process, the thin film sample was exposed by the fundamental beam and its $\mathrm{SH}$ simultaneously. After the poling process the seeding $\mathrm{SH}$ was cut by a color filter and the photoinduced $\chi^{(2)}$ ws probed by the fundamental beam. The detailed description of the experimental setup can be found elsewhere [7].

The poling effect at a given point of the sample strongly depends on the relative phase of $\omega$ and $2 \omega$, if the thickness of the sample is much smaller than the coherent length $l_{\mathrm{c}}=2 \pi /\left(k_{2 \omega}-2 k_{\omega}\right)=$ $\lambda_{\omega} / 2 /\left(n_{2 \omega}-n_{\omega}\right)$. We calculated the refractive index difference $\Delta n=n_{2 \omega}-n_{\omega}$ from the absorption spectra using Kramers-Kronig's relation and found it to be $\sim 0.04$ for both the monomer and polymer samples. This gives the coherent length about $12 \mu \mathrm{m}$. So, it is important to bear in mind that the phase difference $\Delta \phi=\phi_{2 \omega}-2 \phi_{\omega}$ in the interference term can be varied when carrying out experiments. The final saturated level of $\chi^{(2)}$ also depends on ratio between polar and non-polar terms. Fiorini et al. [2] have demonstrated that large enhancement of photoinduced $\chi^{(2)}$ can be obtained by optimizing of the ratio between $\omega$ and $2 \omega$ seeding intensities.

Fig. 2 presents the intensity dependence of the photoinduced $\chi^{(2)}$ in the substituted norbornene and DR1-PMMA samples. In this experiment seeding intensities were optimized only in one point (about $0.3 \mathrm{GW} / \mathrm{cm}^{2}$ and $3 \mathrm{~kW} / \mathrm{cm}^{2}$ for fundamental and $\mathrm{SH}$, respectively) for the DR1-

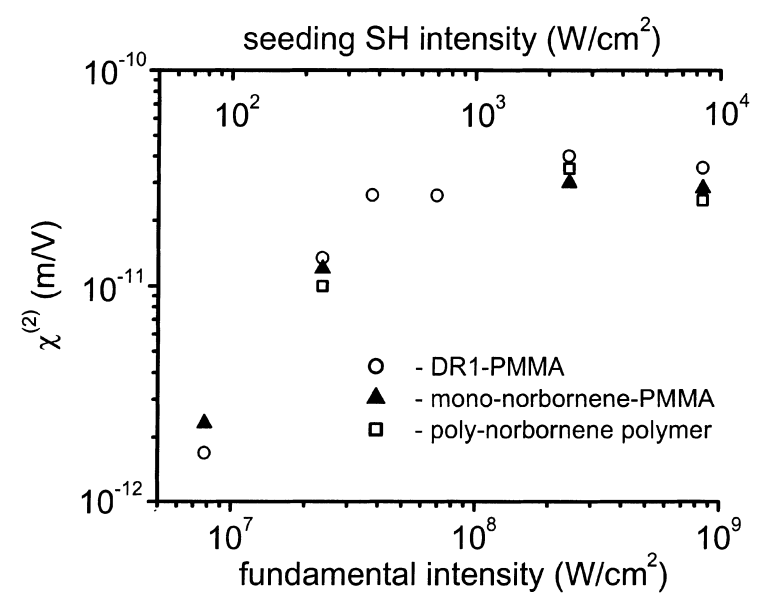

Fig. 2. Photoinduced $\chi^{(2)}$ as a function of seeding intensities presented in the log-log scale.

PMMA sample, and further the intensities of input beams were varied in the wide range (about $0.01-1 \mathrm{GW} / \mathrm{cm}^{2}$ for fundamental and $0.1-10 \mathrm{~kW} / \mathrm{cm}^{2}$ for $\mathrm{SH}$ ) by changing the sample position relative to the focal point. In each position the sample was seeded for $40 \mathrm{~min}$. After seeding process the external $\mathrm{SH}$ was blocked by a color filter and the photoinduced $\mathrm{SH}$ generation was detected by a photomultiplier tube (PMT). The $\chi^{(2)}$ value was calculated according to Ref. [2] taking into account absorption and dispersion of the samples. The relative phase $\Delta \phi$ was optimized for each point in Fig. 2 by tuning a glass plate put before the focusing lens. The aim of this study is to compare optical poling efficiency of the substituted norbornene and DR1-PMMA samples in wide range of intensities (for the time being, without optimizing of poling intensities, as was done in [2]). Fig. 2 shows the $\chi^{(2)}$ induced in both monoand poly-norbornene samples are close to that in the DR1-PMMA and at low intensities the $\chi^{(2)}$ of the monomer sample even exceeds it. The $\chi^{(2)}$ is almost not changed at the intensities above $30 \mathrm{MW} / \mathrm{cm}^{2}$ of the fundamental and $300 \mathrm{~W} / \mathrm{cm}^{2}$ of the SH. This is probably due to the saturation of the molecular orientation. Indeed, the maximum $\chi^{(2)}$ value obtained in the mono-norbornene sample $\left(30 \mathrm{pm} / \mathrm{V}=6 \times 10^{-7} \mathrm{esu}\right)$ is about $0.27 f N \beta$, where $N=1.95 \times 10^{20} \mathrm{~cm}^{-3}$ is the concentration of chromophores, $\beta=4.16 \times 10^{-27}$ esu 
is their first hyperpolarizability [5] and $f \approx 2.7$ is a collection of local field factors [8]. This value is close to the theoretical value of the saturated $\chi^{(2)}\left(\chi^{(2)} \approx(1 / 4) f N \beta[4]\right)$, if the orientational diffusion and the cis population life-time is neglected.

Fig. 2 also shows that considerable molecular orientation can be achieved at relatively low seeding intensities even without special optimization of the ratio between them. Another important consequence of the data presented in Fig. 2 is that the norbornene polymer sample does not reveal any significant enhacnement of $\chi^{(2)}$ compared to the monomer sample, despite a higher concentration of the chromophores $\left(N \sim 1.5 \times 10^{21} \mathrm{~cm}^{-3}\right)$ and a previously demonstrated considerably higher $\beta$ value of the polymer molecule in comparison with the monomer one [5]. This is due to the rigidity of the poly-norbornene polymer backbone and the strong orientational correlation between pendant chromophores that reduce reorientation of molecules.

Fig. 3 depicts the evolution of the photoinduced $\chi^{(2)}$ during optical poling process in the DR1PMMA and norbornene monomer samples. To avoid distortion of the growth curves by frequent interruption of the poling process for probing, we applied simultaneous writing and probing technique described in Ref. [7]. This method proved itself to be more accurate in comparison with traditional alternating writing and probing technique [7]. The seeding intensities were about $0.6 \mathrm{GW} / \mathrm{cm}^{2}$ of the fundamental and $3 \mathrm{~kW} / \mathrm{cm}^{2}$ of the $\mathrm{SH}$ for both samples. The growth curves were fitted by a bi-exponential function

$f(t)=B_{1}\left(1-\exp \left(-t / \tau_{B_{1}}\right)\right)+B_{2}\left(1-\exp \left(-t / \tau_{B_{2}}\right)\right)$.

This fitting function was chosen because of earlier demonstrated bi-exponential $\chi^{(2)}$ decay [4,9], which probably is preceded by a bi-exponential buildup of $\chi^{(2)}$. It should be noted that time constants $\tau_{B_{1}}$ and $\tau_{B_{2}}$ for the mono-norbornene sample are several times larger than those for DR1-PMMA (Fig. 3), which is due to the smaller absorption of the norbornene sample at $532 \mathrm{~nm}$ wavelength. On the other hand, the parameter $B_{1}$ and $B_{2}$ are close to each other for both DR1-PMMA
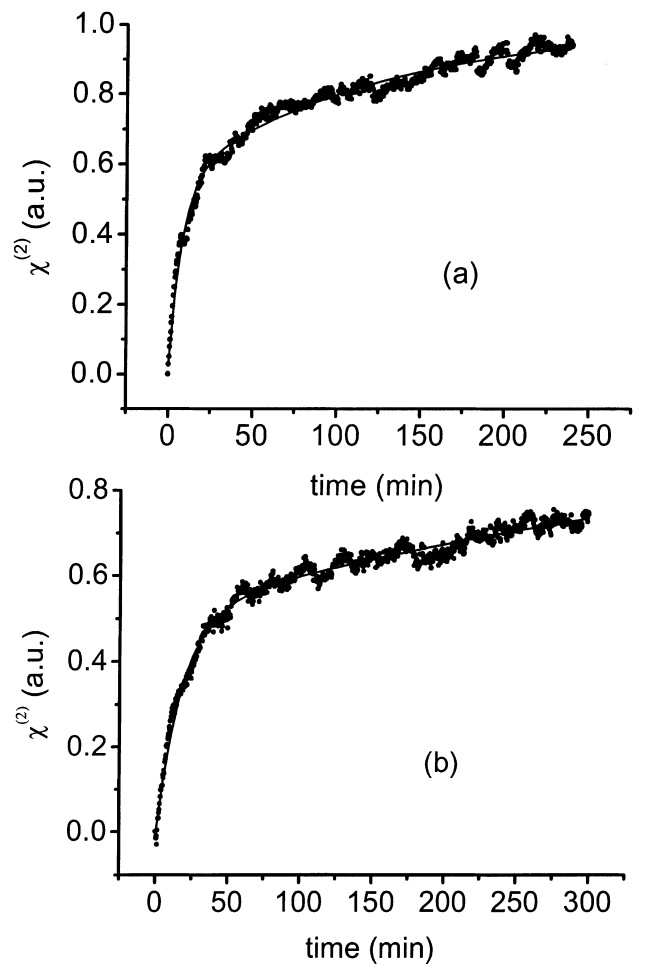

Fig. 3. Photoinduced $\chi^{(2)}$ as a function of time during optical poling process in DR1-PMMA sample (a) and mono-norbornene-PMMA sample (b). Solid cruves are the fits to a two-exponential function $f(t)=B_{1}\left(1-\exp \left(-t / \tau_{B_{1}}\right)\right)+B_{2}(1-$ $\left.\exp \left(-t / \tau_{B_{2}}\right)\right)$. The fitting parameters are $\tau_{B_{1}}=8 \mathrm{~min}, \tau_{B_{2}}=126$ $\min , B_{2} / B_{1}=0.87$ for the DR1-PMMA sample and $\tau_{B_{1}}=18$ $\min , \tau_{B_{2}}=520 \mathrm{~min}, B_{2} / B_{1}=0.72$ for the mono-norbornene sample.

$\left(B_{2} / B_{1}=0.87\right)$ and mono-norbornene sample $\left(B_{2} / B_{1}=0.72\right)$. However, it was a problem to apply the simultaneous writing and probing techniques to the poly-norbornene sample because of the relatively weak SH signal that is due to small thickness of the polymer sample. The $\chi^{(2)}$ growth measurement in poly-norbornene sample with the alternating writing and probing gives the same order of the $\chi^{(2)}$ buildup rate as in the monomer sample, but more precise comparison of the fit parameters is difficult, because, in the case of polymer sample, the induced $\chi^{(2)}$ partially decays during stops in seeding [7].

The non-exponential growth dynamics is explained as follows. In first few seconds of poling, the angular hole burning in the molecular 
distribution occurs because of different excitation probability for molecules with parallel and antiparallel orientation with respect to the polar axis. After excitation the molecules tend to relax to the cis-state with a smaller first hyperpolarizability, so the initial short-lived macroscopic $\chi^{(2)}$ emerges. Reverse thermal cis-trans relaxation can return the molecule to the original trans-state or to the transstate with a different orientation. Finally, the molecule tends to be reoriented to the state with minimum excitation probability. The reorientation rate obviously depends on the excitation probability and, therefore, on the angle $\theta$ between polar axis and the dipole moment of the molecule. Analysis of the terms that contribute to the total excitation probability $P(\theta)$ shows that $P(\theta)$ can considerably differ for the molecules originally oriented at the angles $\theta<\pi / 2$ and $\pi / 2<\theta<\pi$. Therefore, the time constants $\tau_{B_{2}}$ and $\tau_{B_{1}}$ can be attributed to the reorientation of the molecules originally oriented at the angles $\theta<\pi / 2$ and $\pi / 2<\theta<\pi$, respectively.

Fig. 4 shows the decay of the $\chi^{(2)}$ induced in DR1-PMMA and substituted norbornene monomer and polymer samples after $5 \mathrm{~s}$ (a) and $40 \mathrm{~min}$ (b) of poling. The solid lines are fits to a bi-exponential function $f(t)=A_{0}+A_{1} \exp \left(-t / \tau_{A_{1}}\right)+A_{2} \exp (-t /$ $\tau_{A_{2}}$ ) with $\tau_{A_{1}} \sim 15 \mathrm{~s}$ and $\tau_{A_{2}} \sim 120 \mathrm{~s}$ (Fig. 4a) and a mono-exponential function $f(t)=A_{0}+A \exp (-t /$ $\left.\tau_{A}\right)$ with $\tau_{A} \sim 500 \mathrm{~s}$ (Fig. $4 \mathrm{~b}$ ). The fit parameters are
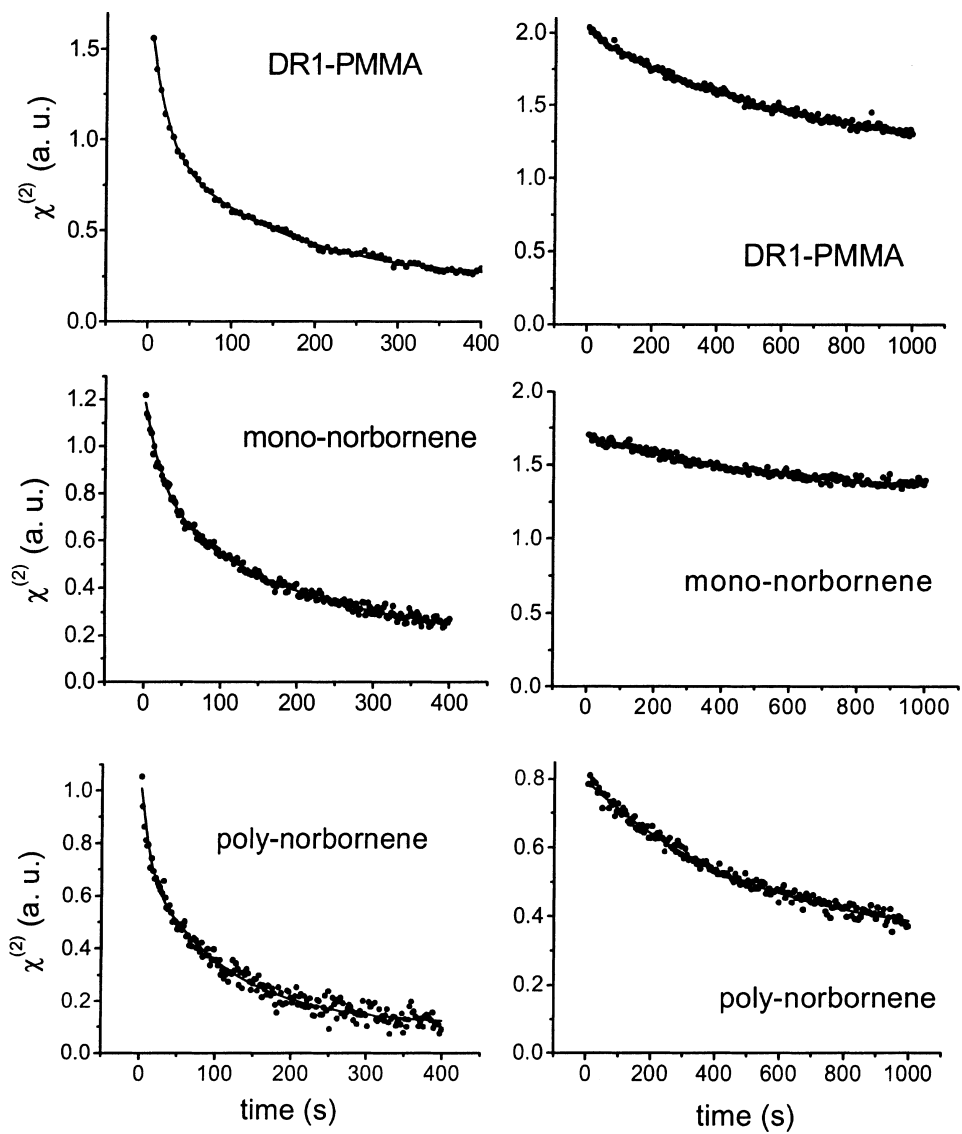

(a)

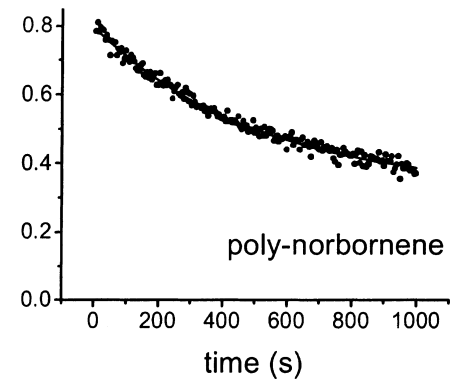

(b)

Fig. 4. Decay of $\chi^{(2)}$ after $5 \mathrm{~s}$ (a) and $40 \mathrm{~min}$ (b) of optical poling. The experimental data are fitted to a bi-exponential function $f(t)=A_{0}+A_{1} \exp \left(-t / \tau_{A_{1}}\right)+A_{2} \exp \left(-t / \tau_{A_{2}}\right)$ (a) and to a mono-exponential function $f(t)=A_{0}+A \exp \left(-t / \tau_{A}\right)(\mathrm{b})$. 
Table 1

Fit parameters in the functions $f(t)=A_{0}+A_{1} \exp \left(-t / \tau_{A_{1}}\right)+A_{2} \exp \left(-t / \tau_{A_{2}}\right)$ and $f(t)=A_{0}+A \exp \left(-t / \tau_{A}\right)$ used in Fig. $4^{\mathrm{a}}$

\begin{tabular}{|c|c|c|c|c|c|c|c|c|}
\hline \multirow[t]{2}{*}{ Sample } & \multicolumn{5}{|c|}{ After $5 \mathrm{~s}$ poling } & \multicolumn{3}{|c|}{ After $40 \mathrm{~min}$ poling } \\
\hline & $\begin{array}{l}A_{0} \\
\text { (au) }\end{array}$ & $\begin{array}{l}A_{1} \\
\text { (au) }\end{array}$ & $\begin{array}{l}A_{2} \\
\text { (au) }\end{array}$ & $\begin{array}{l}\tau_{A_{1}} \\
\text { (s) }\end{array}$ & $\begin{array}{l}\tau_{A_{2}} \\
(\mathrm{~s})\end{array}$ & $\begin{array}{l}A_{0} \\
\text { (au) }\end{array}$ & $\begin{array}{l}A \\
(\mathrm{au})\end{array}$ & $\begin{array}{l}\tau_{A} \\
(\mathrm{~s})\end{array}$ \\
\hline DR1 & 0.22 & 0.78 & 0.77 & 17 & 146 & 1.16 & 0.84 & 569 \\
\hline Mono-norbornene & 0.19 & 0.41 & 0.62 & 21 & 167 & 1.31 & 0.39 & 501 \\
\hline Poly-norbornene & 0.1 & 0.36 & 0.61 & 10 & 110 & 0.32 & 0.47 & 513 \\
\hline
\end{tabular}

${ }^{\mathrm{a}}$ The parameters were obtained by successive approximations with Microcal Origin Software. The relative uncertainties for any parameter do not exceed $6 \%$.

summarized in Table 1 . The constant $A_{0}$ represents the part of $\chi^{(2)}$ that is due to quasi-permanent net orientation with life-time much larger than $\tau_{A}$. The multi-exponential decay after $5 \mathrm{~s}$ poling can be explained by relaxation of angular hole burning due to reverse cis-trans transition (fast component) and disorientation of trans-molecules caused by thermal motion and reorientation by non-polar fundamental radiation (slow component). After long poling time (40 $\mathrm{min}$ ), the $\chi^{(2)}$ is mainly due to molecular net orientation, therefore, it takes longer time to destroy the polar order.

We also studied the dark relaxation of $\chi^{(2)}$ by switching on the probing beam only for short periods of time. No considerable difference in $\chi^{(2)}$ decay rate has been found for dark relaxation and the relaxation in the presence of probing beam, therefore, the decrease of $\chi^{(2)}$ after 40 min poling is mainly due to dark relaxation.

One can see in Fig. 4b, that the $\chi^{(2)}$ in the monomer norbornene sample is more stable than those in DR1-PMMA and poly-norbornene samples. The relative weight of the quasi-permanent component $A_{0}$ of the monomer sample is about two times higher than that of the polymer one. This can be also explained by the rigidity of the polymer backbone and the strong orientational correlation between side-chain chromophores, which reduce free rotation of the attached chromophores and the whole backbone. Indeed, the molecular reorientation in a solid polymer matrix is possible because the molecule receives some angular momentum as a result of the thermal relaxation. After many trans-cis-trans cycles, the molecule finally achieves the orientation with a minimal excitation probability. This mechanism is effective for a azo-dye guest-host system or for polymers, in which an attached chromophore is allowed to rotate regardless the state of the other molecules (e.g., DR1-MMA copolymer [2]). However, in the poly-norbornene sample, the chromophores are all perpendicularly attached to the polymer backbone (see Fig. 1) to form a rigid orientationally correlated system. Since the number of the attached molecules $n_{\mathrm{c}} \gg 1$ and reorientation of each individual molecule is random, the total orientational effect for the whole polymer unit is strongly reduced. Therefore, there is no considerable enhancement of the $\chi^{(2)}$ despite the high first hyperpolarizability value shown in the HRS experiment [5]. This is in contrast with the previous results on DR1-PMMA side-chain copolymer, which possess a flexible backbone and revealed remarkable enhancement of $\chi^{(2)}$ compared to guest-host DR1-PMMA system [2].

It is also important that within a given polymer unit all the side-chain molecules are uniformly distributed in the $120^{\circ}$ angular range [5]. Therefore, the excitation probability for individual side-chain molecules can greatly vary. This results in selective reorientation of side-chain molecules with higher excitation probability and arising elastic tensions in the polymer backbone. After the optical poling field is switched off, the backbone tends to return the molecules back to the states with their original orientations. This can be a reason for the faster decay of the SH signal in the poly-norbornene sample compared to the mono-norbornene one.

In conclusion, we have demonstrated efficient optical poling of substituted norbornene polymer thin films. Considerable $\chi^{(2)}$ value (several $\mathrm{pm} / \mathrm{V}$ ) can be achieved at low seeding intensities (about $10 \mathrm{MW} / \mathrm{cm}^{2}$ for the fundamental and $100 \mathrm{~W} / \mathrm{cm}^{2}$ for the $\mathrm{SH}$ of a Q-switched Nd:YAG laser). We 
have also found that no appreciable enhancement of encoded $\chi^{(2)}$ can be obtained in the thin film of poly-norbornene polymer compared to the mononorbornene thin film. The result is in contrast to the case of DR1-MMA co-polymer, which revealed a large enhancement of the $\chi^{(2)}$ compared to $10 \%$ DR1-doped PMMA. This difference is explained by the rigidity of the poly-norbornene polymer backbone and the strong orientational correlation between pendant chromophores that reduce the reorientation effect.

\section{Acknowledgements}

The authors gratefully acknowledge the financial supports from the National Science Council and Academia Sinica of Taiwan. The research of V.M. Churikov was also supported by Institute of Chemistry of the Academia Sinica.

\section{References}

[1] L.M. Hayden, G.F. Sauter, F.R. Ore, P.L. Pasillas, J.M. Hoover, G.A. Lindsay, R.A. Henry, J. Appl. Phys. 58 (1990) 456.

[2] C. Fiorini, F. Charra, J.M. Nunzi, P. Raimond, J. Opt. Soc. Am. B 14 (1997) 1984.

[3] C. Fiorini, J.M. Nunzi, Chem. Phys. Lett. 286 (1998) 415.

[4] G. Xu, X. Liu, J. Si, P. Ye, Z. Li, Y. Shen, Opt. Lett. 25 (2000) 329.

[5] C.C. Hsu, T.H. Huang, S. Liu, F.F. Yeh, B.Y. Jin, J.A. Sattigeri, C.W. Shiau, T.Y. Luh, Chem. Phys. Lett. 311 (1999) 355.

[6] J.A. Sattigeri, C.W. Shiau, C.C. Hsu, F.F. Yeh, S. Liu, B.Y. Jin, T.Y. Luh, J. Am. Chem. Soc. 121 (1999) 1607.

[7] V.M. Churikov, M.F. Hung, C.C. Hsu, Opt. Lett. 25 (2000) 960.

[8] P.N. Prasad, D.J. Williams, Introduction to Nonlinear Optical Effects in Molecules and Polymers, Wiley, New York, 1991.

[9] G. Xu, J. Si, X. Liu, Q.G. Yang, P. Ye, J. Appl. Phys. 85 (1999) 681. 